\title{
Alteration of intra-pancreatic target-organ specificity by abrogation of Aire in NOD mice
}

\author{
Shino Niki, ${ }^{1}$ Kiyotaka Oshikawa, ${ }^{1}$ Yasuhiro Mouri, ${ }^{1}$ Fumiko Hirota, ${ }^{1}$ Akemi Matsushima, ${ }^{1}$ \\ Masashi Yano, ${ }^{1}$ Hongwei Han, ${ }^{1}$ Yoshimi Bando,2 Keisuke Izumi,2 Masaki Matsumoto, 3,4 \\ Keiichi I. Nakayama, ${ }^{3,4}$ Noriyuki Kuroda, ${ }^{1}$ and Mitsuru Matsumoto'
}

\author{
1Division of Molecular Immunology, Institute for Enzyme Research, University of Tokushima, Tokushima, Japan. \\ 2Department of Molecular and Environmental Pathology, Institute of Health Biosciences, University of Tokushima Graduate School, Tokushima, Japan. \\ ${ }^{3}$ Department of Molecular and Cellular Biology, Medical Institute of Bioregulation, Kyushu University, Fukuoka, Japan. \\ ${ }^{4}$ Core Research for Evolutional Science and Technology, Japan Science and Technology Corp., Saitama, Japan.
}

\begin{abstract}
Factors that determine the spectrum of target organs involved in autoimmune destruction are poorly understood. Although loss of function of autoimmune regulator (AIRE) in thymic epithelial cells is responsible for autoimmunity, the pathogenic roles of AIRE in regulating target-organ specificity remain elusive. In order to gain insight into this issue, we have established NOD mice, an animal model of type 1 diabetes caused by autoimmune attack against $\beta$ cell islets, in which Aire has been abrogated. Remarkably, acinar cells rather than $\beta$ cell islets were the major targets of autoimmune destruction in Aire-deficient NOD mice, and this alteration of intra-pancreatic target-organ specificity was associated with production of autoantibody against pancreas-specific protein disulfide isomerase (PDIP), an antigen expressed predominantly by acinar cells. Consistent with this pathological change, the animals were resistant to the development of diabetes. The results suggest that Aire not only is critical for the control of self-tolerance but is also a strong modifier of target-organ specificity through regulation of $T$ cell repertoire diversification. We also demonstrated that transcriptional expression of PDI $p$ was retained in the Aire-deficient NOD thymus, further supporting the concept that Aire may regulate the survival of autoreactive $T$ cells beyond transcriptional control of self-protein expression in the thymus.
\end{abstract}

\section{Introduction}

IDDM results from autoimmune destruction of insulin-producing pancreatic $\beta$ cells $(1,2)$. The nature of immune dysregulation leading to $\beta$ cell destruction remains poorly understood, but it is clearly influenced by multiple genetic, environmental, and immunological factors. The NOD mouse is a widely used animal model of IDDM, sharing major characteristics with the human disease $(3,4)$. Multiple genetic loci that control disease susceptibility have been mapped (5). Experimentally, defective central tolerance has been implicated in at least part of the pathogenic process in NOD mice, and this defective tolerance appears to be caused by an intrinsic defect in the apoptosis process in NOD thymocytes during negative selection (6-8). However, since establishment of self-tolerance primarily depends on physical contact between thymocytes and thymic stroma (9), it is also important to characterize stromal factors that might regulate the diabetic process in NOD mice.

Mutation of the autoimmune regulator (AIRE) gene is responsible for the development of an organ-specific autoimmune disease (autoimmune polyendocrinopathy-candidiasis-ectodermal dystrophy [APECED]) that demonstrates monogenic autosomal recessive inheritance $(10,11)$. As expected, deletion of the Aire gene in mice also results in the development of organ-specific autoimmune disease, although there are differences in target-organ specificity between human patients and Aire-deficient mice (12-14). Because medullary thymic epithelial cells (TECs) play pivotal roles in the cross-

Nonstandard abbreviations used: AIRE, autoimmune regulator; APECED, autoimmune polyendocrinopathy-candidiasis-ectodermal dystrophy; CY, cyclophosphamide; PD-1, programmed cell death 1; PDIp, pancreas-specific protein disulfide isomerase; TEC, thymic epithelial cell; UEA-1, Ulex europaeus agglutinin 1.

Conflict of interest: The authors have declared that no conflict of interest exists. Citation for this article: J. Clin. Invest. 116:1292-1301 (2006). doi:10.1172/JCI26971. talk between developing thymocytes and thymic stroma (15), and Aire-deficient TECs show reduced transcription of a group of genes encoding peripheral antigens $(13,14,16)$, it is reasonable to speculate that pathogenic autoreactive $\mathrm{T}$ cells escape negative selection because of reduced expression of the corresponding target antigens in the Aire-deficient thymus $(13,17)$. However, other mechanisms of Aire-dependent tolerance also remain possible. Indeed, we have demonstrated that Aire-deficient mice develop autoimmunity against a transcriptionally unrepressed target antigen in the thymus (14). We therefore speculated that Aire might additionally regulate the processing and/or presentation of self-proteins so that maturing $\mathrm{T}$ cells can recognize self-antigens in a form capable of efficiently triggering autoreactive $T$ cells. This alternative view of the function of Aire in the establishment of central tolerance has recently been supported by a study with transgenic mice expressing a model antigen under the control of a tissue-specific promoter together with a TCR specific for the corresponding antigen in the absence of Aire (18).

An important aspect of the study of autoimmune disease is target-organ specificity. Clearly, $\beta$ cell islets are the predominant target of autoimmune attack in NOD mice, although the exact molecule(s) recognized by autoreactive $T$ cells is still under debate (19). Similarly, autoimmune attack is mostly confined to the exocrine organs, such as salivary and lacrimal glands, in our Aire-deficient mice, except for the additional development of gastritis in mice on a $\mathrm{BALB} / \mathrm{c}$ background (14). It remains largely unknown how target-organ specificity is defined by the NOD mouse background or by Aire deficiency in these animals.

In order to gain further insights into the contribution of AIRE to the establishment of central tolerance, as well as the pathogenic roles of AIRE in regulating target-organ specificity, we have established NOD mice lacking Aire. Aire-deficient NOD mice demonstrated both expected and unexpected autoimmune phenotypes. As 
A

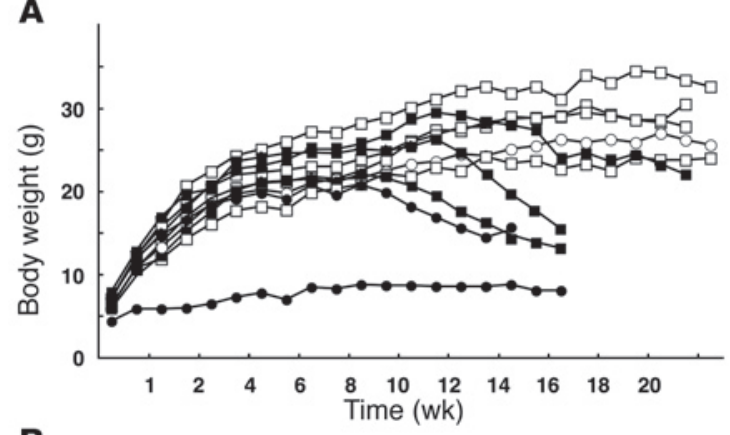

B

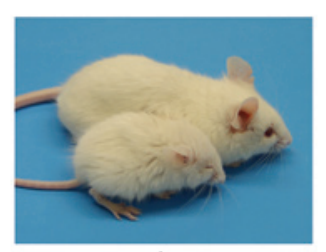

C

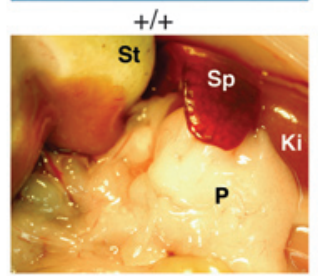

$-1-$
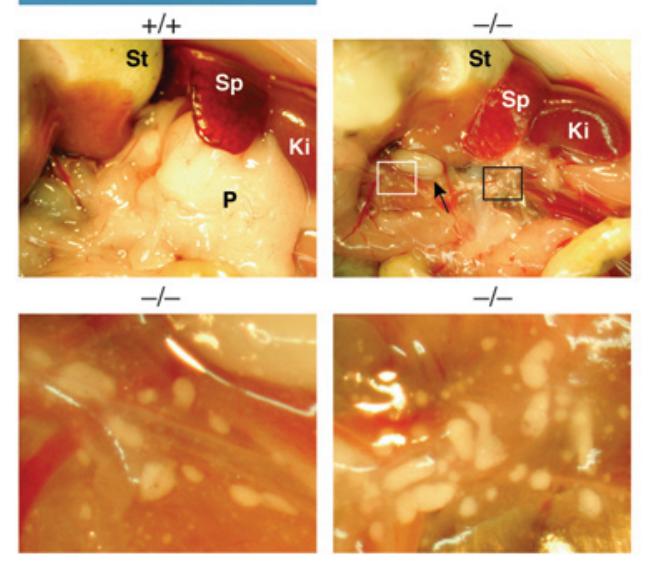
$-1-$

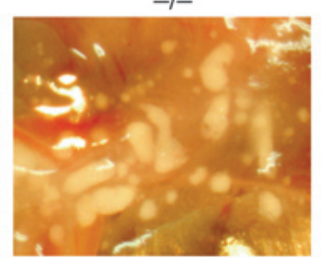

expected, we found obviously augmented autoimmune disease with early onset, which may be explained by the combined effect of the intrinsic NOD T cell defect together with the stromal abnormality resulting from lack of Aire, both of which affect central tolerance. Unexpectedly, we found resistance to the development of overt diabetes in Aire-deficient NOD mice, and this resulted from a change in the target cells attacked by the autoreactive $\mathrm{T}$ cells; acinar cells rather than $\beta$ cell islets were the major targets of autoimmune destruction in Aire-deficient NOD mouse pancreas. Consistent with this alteration of intra-pancreatic target-organ specificity, we identified pancreas-specific protein disulfide isomerase (PDIp), a molecule expressed predominantly by acinar cells $(20,21)$, as an autoantigen recognized by Aire-deficient NOD mouse serum. Polyclonal B cell activation in Aire-deficient NOD mice was another unexpected feature in light of the fact that both Aire deficiency and NOD mice are considered to be models of organ-specific autoimmunity rather than systemic autoimmunity. Our studies with Aire-deficient mice on an autoimmune-prone NOD mouse background highlight novel aspects of Aire in the pathogenesis of autoimmune disease.

\section{Results}

Augmented autoimmune phenotypes in NOD mice lacking Aire. We have recently demonstrated that Aire-deficient mice develop Sjögren syndrome-like pathological changes in their exocrine organs (14). With the use of inbred Aire-deficient mouse strains, we have also demon-

\section{Figure 1}

Growth abnormalities and pancreatic atrophy in Aire-deficient NOD mice. (A) Body weights of individual female (circles) and male (squares) Aire-sufficient (open symbols) and Aire-deficient (filled symbols) mice after birth were plotted. Measurement for many Aire-deficient NOD mice was terminated around 15-17 weeks after birth because of their lethal phenotypes. (B) Growth retardation of Aire-deficient NOD mice. Littermates of 7-week-old males are shown. (C) Pancreatic mass was absent from many Aire-deficient NOD mice upon gross inspection. Instead, many small white patches were scattered throughout the thin and lucent adipose tissues. The square areas marked with white and black lines are enlarged and shown in the bottom left and bottom right panels, respectively. An arrow (in the top right panel) indicates an enlarged pancreatic lymph node. Original magnification, $\times 1.2$. Ki, kidney; P, pancreas; Sp, spleen; St, stomach.

strated the presence of some additional factor(s) that determines the target-organ specificity of the autoimmune disease caused by Aire deficiency; Aire-deficient BALB/c mice, but not Aire-deficient C57BL/6 mice, additionally show lymphoid cell infiltration in the gastric mucosa (14). In turn, we asked whether NOD mice lacking Aire might develop augmented autoimmune phenotypes and/or distinct target-organ specificity compared with NOD mice possessing Aire. We backcrossed our original strain of Aire-deficient mice to NOD mice for 6-9 generations. Heterozygous Aire-deficient mice were crossed to obtain homozygous Aire-deficient NOD offspring. Offspring homozygous for Aire deficiency were recognized in numbers slightly lower than expected from the heterozygous crossing when assessed 4 weeks after birth: Aire $^{+/+} /$Aire $^{+/-} /$Aire $^{-/-}=64: 111: 44$ (mice backcrossed onto NOD for 7-9 generations were subjected to the analysis). Remarkably, many Aire-deficient NOD mice exhibited body-weight loss starting from 8-12 weeks after birth, as revealed by monitoring of 1 group of littermates chosen randomly (Figure 1A). More strikingly, one-third of Aire-deficient NOD mice (11 of 27 females and 8 of 30 males) demonstrated marked growth retardation from the neonatal stage (i.e., less than $50 \%$ of the body weight of their control littermates) with a gaunt appearance (Figure 1, A and B). Upon gross inspection of the organs, we noticed reduction of pancreas size (including the fat tissues surrounding the pancreas) in most of the Aire-deficient NOD mice, or even almost complete loss of pancreatic mass in one-third of the animals (Figure 1C, top right panel). In the latter animals, we observed many white patches 1-2 mm in size scattered throughout the thin and lucent adipose tissues (Figure 1C, bottom panels). These patches consisted of $\beta$ cell islets, pancreatic ducts, and marked lymphoid cell infiltrations (see below). Pancreatic lymph nodes (Figure 1C, top right panel) together with other peripheral lymph nodes, such as submandibular and axillary lymph nodes (data not shown), were enlarged in many animals.

Upon histological evaluation, we observed marked lymphoid cell infiltration in various organs from Aire-deficient NOD mice, with 2 particular characteristics. First, the degree of lymphoid cell infiltration of the organs affected in the original (i.e., Aire-sufficient) NOD mice was much more severe in Aire-deficient NOD mice. Most pancreatic islets from many Aire-deficient NOD mice were surrounded by massive lymphoid cell infiltration, as if the $\beta$ cell islets were floating on a sea of lymphoid cells (Figure 2A, bottom left panel). Furthermore, in many cases, the massive lymphoid cell infiltration completely destroyed acinar structures, leaving relatively well-preserved $\beta$ cell islets together with pancreatic ducts densely surrounding the $\beta$ cell islets alone (Figure $2 \mathrm{~A}$, middle panels). The degree of lymphoid cell infiltration in the salivary glands, which occurs in the original 
A

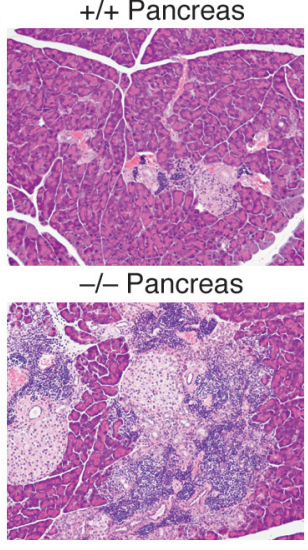

B

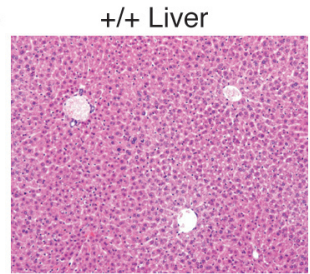

-1- Liver

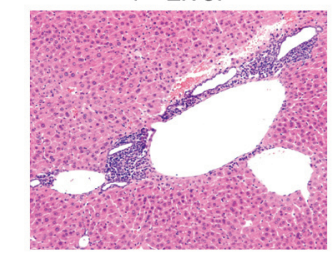

C $+/+T$ cell transferred

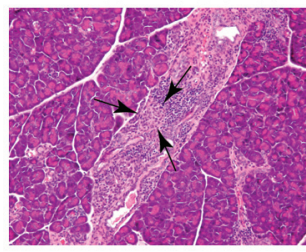

-/- Pancreas

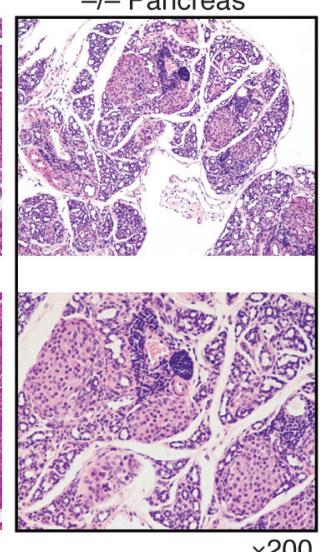

$\times 200$

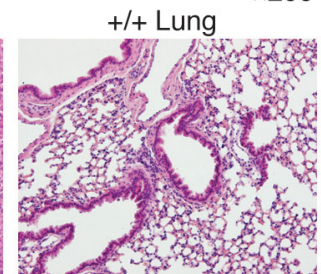

-l- Lung

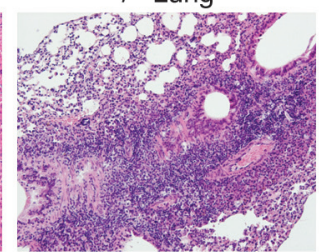

-l- T cell transferred

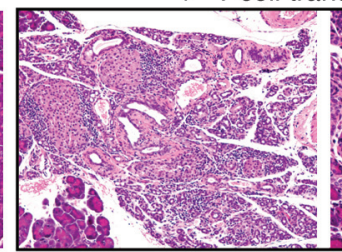

$+/+$ Salivary gland

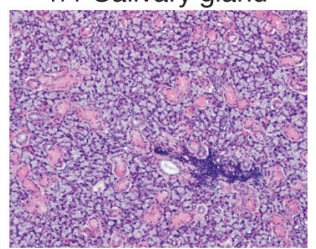

-1- Salivary gland

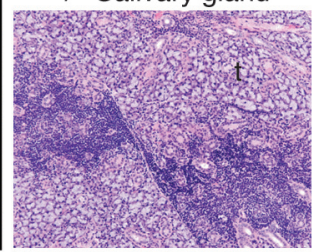

+ +t Thyroid

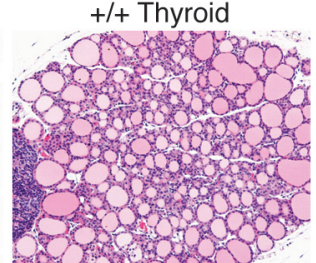

-1- Thyroid
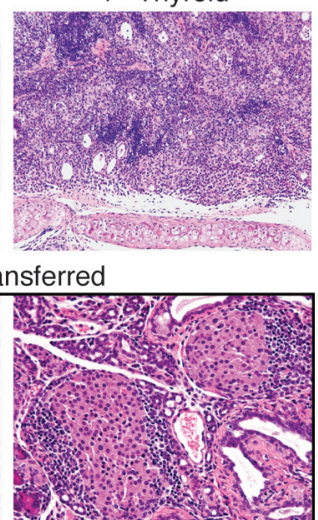

\section{Figure 2}

Augmented autoimmune phenotypes with altered intrapancreatic target-organ specificity in Aire-deficient NOD mice. (A) Augmentation of existing autoimmune phenotypes of NOD mice by abrogation of Aire. In Aire-deficient NOD mice, lymphocytic infiltration in the pancreas was much more severe than that in control littermates (left panels). In many Aire-deficient NOD mice, acinar tissues were completely destroyed by marked lymphocytic infiltration, leaving relatively well-preserved $\beta$ cell islets together with pancreatic ducts densely surrounding the $\beta$ cell islets alone (middle panels). The 2 boxed panels are photographs taken of the same sample with different magnifications. Sialoadenitis was also much more severe in Aire-deficient NOD mice (right panels). (B) Lymphoid cell infiltration in the liver, lung, and thyroid gland from Aire-deficient NOD mice. (C) NOD-scid mice transferred with mature T cells purified from Aire-deficient NOD mice showed lymphocytic infiltration predominantly in acinar tissues, and the structure of the $\beta$ cell islets was relatively well preserved (middle and right panels). In contrast, NOD-scid mice transferred with Aire-sufficient NOD mouse T cells showed lymphocytic infiltration into $\beta$ cell islets, resulting in reduced size and numbers of $\beta$ cell islets (left panel). Arrows indicate the 1 small $\beta$ cell islet remaining. Original magnification, $\times 100$, except where indicated.
NOD mice, was also much more severe in Aire-deficient NOD mice (Figure 2A, right panels). Secondly, we observed lymphoid cell infiltration in many other organs of Aire-deficient NOD mice, including liver, lung, and thyroid gland (Figure $2 \mathrm{~B}$ ) and prostate and seminal vesicle in males (Supplemental Table 1 and data not shown; supplemental material available online with this article; doi:10.1172/ JCI26971DS1), in which we usually do not observe changes in the original NOD mice. Thus, abrogation of Aire in NOD mice resulted in expansion of the spectrum of target organs destroyed by autoimmune attack together with an alteration of intra-pancreatic targetorgan specificity from $\beta$ cell islets to acinar cells (see below). The growth abnormalities seen in Aire-deficient NOD mice described above might be partially explained by the digestive problems caused by loss of the exocrine pancreas, which has also been suggested in a recent study of Aire-deficient NOD mice (22).

Total spleen cell numbers were indistinguishable between Airedeficient NOD mice and control littermates (i.e., Aire-sufficient NOD mice). Flow-cytometric analysis showed similar expression of B220, CD3, CD4, and CD8 in the spleen (data not shown). Small numbers of Aire-deficient NOD mice (i.e., 6 of 33 analyzed) showed reduced numbers of total thymocytes together with reduction of $\mathrm{CD}^{+} \mathrm{CD}^{+} \mathrm{T}$ cells to variable degrees. The pathological significance of this phenomenon is currently unclear. Despite the augmented autoimmune phenotypes in Aire-deficient NOD mice, the percentages of Foxp $3^{+}$Tregs from both thymus and spleen were not altered in the absence of Aire (Figure 3A), as we and others have reported for Aire-deficient mice on non-autoimmune-prone mouse backgrounds $(14,18)$. Expression of other T cell surface markers, such as CD25, CD62L, and CD69, on splenic T cells was also unchanged in Aire-deficient NOD mice (data not shown). In contrast, CD44high (memory/activated) and ICOS-expressing populations of splenic $\mathrm{CD}^{+}$cells were increased in Aire-deficient NOD mice (Figure 3B).

Immunohistochemical analysis of the Aire-sufficient NOD thymus demonstrated indistinguishable numbers and distribution pattern of medullary TECs, as detected by Ulex europaeus agglutinin 1 (UEA-1) and ER-TR5 $\mathrm{mAb}(14,23)$, with patterns of Aire nuclear dots similar to those from wild-type C57BL/ 6 and BALB/c thymus (Supplemental Figure 1, A and B, top panels); the subcellular distribution of Aire nuclear dots within the cell was also unaltered. Abrogation of Aire in NOD mice did not cause any abnormalities of TEC struc- 


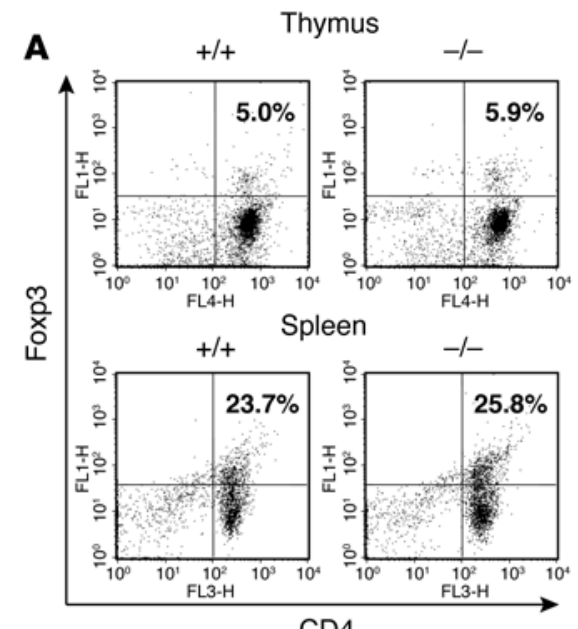

CD4
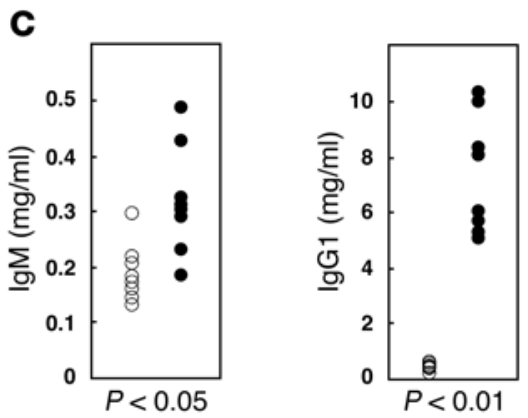

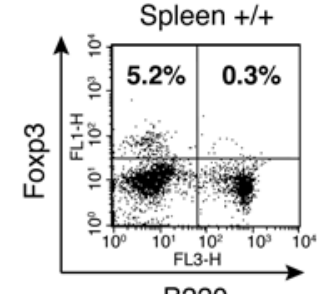

B220
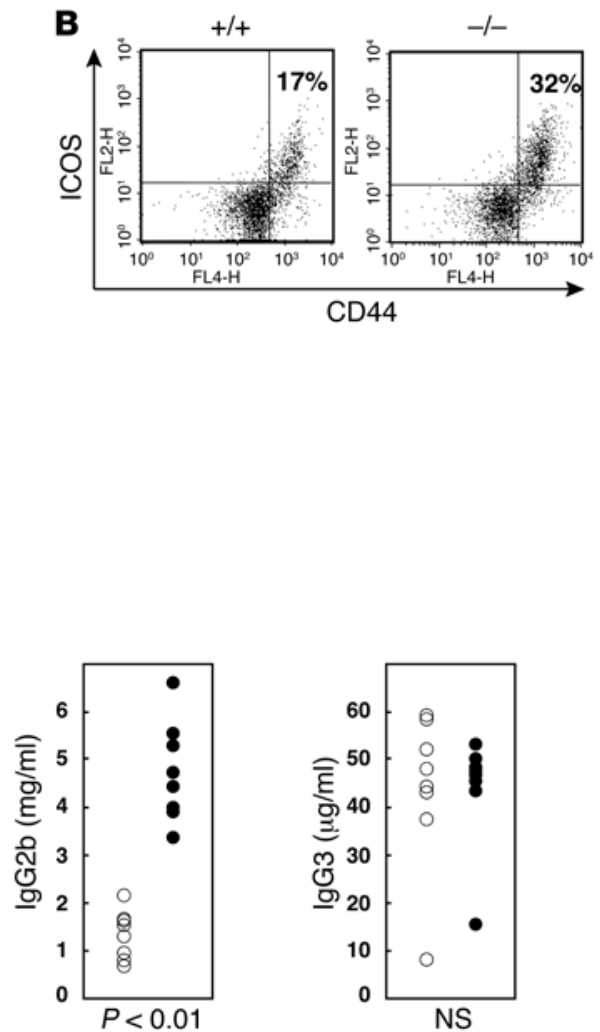

Figure 3

Phenotypic analyses of lymphocytes from Aire-deficient NOD mice. (A) Production of Tregs was retained in Aire-deficient NOD mice. CD3 ${ }^{+}$cells from the thymus and spleen were gated and analyzed for the expression of CD4 and Foxp3. Percentages of CD4 ${ }^{+}$Foxp3 $3^{+}$cells are indicated. In order to verify the specificity of the staining, spleen cells from control mice were stained with anti-Foxp3 mAb. Foxp3 ${ }^{+}$cells were detected only in the B220- cell population, not in the B220+ cell population. (B) Memory/Activated (CD44high) ICOS ${ }^{+}$cells were increased in the spleens from Aire-deficient NOD mice compared with the spleens from control mice. Percentages of CD44high ICOS ${ }^{+}$cells are indicated. CD4 ${ }^{+}$cells were gated and analyzed for CD44 and ICOS expression. (C) Serum Ig levels were elevated in Aire-deficient NOD mice (filled circles) compared with Aire-sufficient NOD littermates (open circles) except for IgG3. One mark corresponds to 1 mouse analyzed.

ture recognized by UEA-1 and ER-TR5 (Supplemental Figure 1A, bottom panels). Expression of several, but not all, of the tissue-specific genes tested, including insulin, in the TECs isolated from Airedeficient NOD mice was reduced, as previously observed in Airedeficient mice of non-autoimmune-prone mouse backgrounds (Table 1) $(13,14,16)$. RNAs extracted from the total thymus of Airedeficient NOD mice showed similar reduced expression of tissuespecific genes, including insulin (Supplemental Table 2).

Alteration of intra-pancreatic target-organ specificity in Aire-deficient NOD mice. Aire-deficient NOD mice started to exhibit lymphoid cell infiltration into the pancreas between 2 and 3 weeks after birth; although these changes were not observed at 1 week after birth ( 0 of 1 female examined), Aire-deficient NOD mice at 2 weeks ( 1 male of 1 female and 1 male) and 3 weeks ( 2 of 2 males) showed peri-insular lymphocytic infiltration (data not shown). In contrast, none of their control littermates showed those changes in the pancreas at less than 3 weeks ( 0 of 2 females and 2 males). This result suggests that abrogation of Aire in NOD mice not only augments the degree of pancreatic lesions once developed, as described above, but also accelerates their onset. Similarly, development of sialoadenitis was accelerated in Aire-deficient NOD mice (S. Niki and M. Matsumoto, unpublished data).

The pancreas is unique in that it functions as both an endocrine and an exocrine organ by secreting insulin as well as several digestive enzymes such as trypsinogen, amylase, and lipase. Careful histological evaluation of the pancreatic lesions in Aire-deficient NOD mice showed unique features of the pathological changes, as described above. The initial change in the pancreas from Aire-deficient NOD mice was perivascular lymphoid cell infiltration near the $\beta$ cell islets, as observed in control littermates (Y. Bando et al., unpublished data). In control mice, lymphoid cell infiltration was directed into $\beta$ cell islets, eventually destroying them and leading to the complete loss of insulin-producing cells (data not shown). In marked contrast, in Aire-deficient NOD mice, although lymphoid cell infiltration became more evident as the mice grew, the histological picture of $\beta$ cell islets being progressively invaded by lymphoid cells was barely apparent (Figure 2A). Instead, acinar tissues surrounding the $\beta$ cell islets were replaced by infiltrating $\mathrm{CD}^{+}, \mathrm{CD}^{+}$, and $\mathrm{B} 220^{+}$ cells (Supplemental Figure 2). In addition to $\mathrm{B}^{2} 20^{+}$cells (Supplemental Figure 2B, top panels), clusters of $\mathrm{CD} 138^{+}$(syndecan- $1^{+}$) cells expressing low levels of B220 were also observed (Supplemental Figure 2B, bottom panels). The outcome of this unusual immune attack in Aire-deficient NOD mice was the complete disappearance of acinar structures, leaving behind relatively well-preserved $\beta$ cell islets and pancreatic ducts densely surrounding the $\beta$ cell islets, which, together with infiltrating lymphoid cells, formed visible white patches throughout the adipose tissues, as demonstrated 


\section{Table 1}

Expression of tissue-specific genes from TECs in Aire-deficient NOD mice

\begin{tabular}{lcccccc} 
Genotype & Foxn1/Hprt & Ins/Hprt & SP1/Hprt & FABP/Hprt & CRP/Hprt & GAD67/Hprt \\
PDIp/Hrpt \\
$+/+$ & 8.10 & $2.54 \times 10^{-1}$ & 6.52 & 6.98 & 4.55 & 30.8 \\
-/- & 10.0 & UD & UD & $9.27 \times 10^{-2}$ & 8.63 & 53.2 \\
Relative abundance (Aire KO/control) & 1.23 & ND & ND & $1 / 75.3$ & 1.90 & 1.59 \\
\hline
\end{tabular}

Real-time PCR for Foxn1 and peripheral tissue-specific genes (Ins, insulin; SP1, salivary protein 1; FABP, fatty acid-binding protein; CRP, C-reactive protein; GAD67, glutamic acid decarboxylase 67; PDIp) was performed using thymic-stroma RNAs from control and Aire-deficient NOD mice. Hprt expression level was used as an internal control. The relative abundance of each gene was calculated from the ratio between the values from control NOD thymus and those from Aire-deficient NOD thymus (e.g., the insulin/Hprt value from Aire-deficient NOD mice was divided by the insulin/Hprt value from control NOD mice). Pools of TECs isolated from 2 mice of each group were used for the analysis. Mice backcrossed onto NOD mice for 6 generations were used. UD, under the limit of detection; ND, not determined.

above (Figures $1 \mathrm{C}$ and $2 \mathrm{~A}$ ). Thus, abrogation of Aire in NOD mice resulted in the dramatic alteration of intra-pancreatic target-organ specificity from endocrine cells toward exocrine cells.

Resistance to diabetes development in Aire-deficient NOD mice. Aire-deficient NOD mice rarely survived more than 20 weeks (Figure 1A), probably because of the severe autoimmune phenotype. By the ages of 12 and 22 weeks, respectively, $1 \mathrm{Aire}^{+/-}$female and $1 \mathrm{Aire}^{+/+}$female had developed overt diabetes, out of 48 control mice $\left(10 \mathrm{Aire}^{+/+}\right.$ females, $12 \mathrm{Aire}^{+/+}$males, $17 \mathrm{Aire}^{+/-}$females, and $9 \mathrm{Aire}^{+/-}$males) monitored for the development of diabetes. In contrast, none of the Airedeficient NOD mice (11 females and 12 males) developed overt diabetes during the same series of observations, which was a predictable consequence of the fact that Aire-deficient NOD mice demonstrated immune attack predominantly against acinar cells rather than $\beta$ cell islets. Absence of hyperglycemia in Aire-deficient NOD mice was not simply caused by feeding problems, since the stomachs of all the Airedeficient NOD mice were found to be full of food when the animals were sacrificed (S. Niki and M. Matsumoto, unpublished data).

In order to evaluate whether Aire-deficient NOD mice are intrinsically resistant to the development of diabetes, we used an induceddiabetes model. We treated mice with cyclophosphamide (CY), an immunosuppressive drug that accelerates diabetic processes in diabetes-prone mice $(24,25)$. Thirteen of 18 control littermates in a total of 3 experiments developed overt diabetes between 11 and 27 days (mean 19.2 days) after the initial injection of CY (Table 2). Although 9 of 11 Aire-deficient NOD mice were unable to tolerate CY treatment and died or were sacrificed between 7 and 22 days (mean 16.8 days) after the initial injection of CY, and only 2 Aire-deficient NOD mice survived throughout this observation period, none of them showed overt diabetes. This result supports the concept that abrogation of Aire in NOD mice results in resistance to development of diabetes, most probably because of alteration of intra-pancreatic target-organ specificity, and this hypothesis was further confirmed by another experiment (see below). A glucose tolerance test performed by i.p. injection of glucose ( $2 \mathrm{mg} / \mathrm{g}$ body weight) into 4 Aire-deficient NOD mice (a 10-week-old male, a 14-week-old female, a 16-week-old male, and a 20-week-old male) also revealed no diabetic pattern in these animals (S. Niki and M. Matsumoto, unpublished data).

Alteration of intra-pancreatic target-organ specificity accounts for the resistance to development of diabetes in Aire-deficient NOD mice. In order to confirm that Aire-deficient NOD mice are resistant to the development of diabetes as a result of alteration of intra-pancreatic targetorgan specificity, we evaluated the disease process by transferring mature T cells isolated from either control littermates or Aire-deficient NOD mice into NOD-scid mice (26). In a first set of experi- ments, 3 of 5 NOD-scid mice transferred with control mouse T cells developed diabetes at 77,120, and 140 days after the transfer (Table 3, experiment 1); 1 NOD-scid mouse transferred with Aire-sufficient

\section{Table 2}

CY-induced diabetes

\begin{tabular}{|c|c|c|c|}
\hline & Genotype & Sex & $\begin{array}{l}\text { Diabetes development } \\
\text { (onset from first dose) }\end{array}$ \\
\hline \multirow[t]{6}{*}{ Exp. $1^{A}$} & $+/+$ & M & Yes (day 27) \\
\hline & $+/+$ & M & No \\
\hline & $+/-$ & M & Yes (day 11) \\
\hline & $-1-$ & M & No \\
\hline & $-1-$ & M & No \\
\hline & $-1-$ & M & ND (sacrificed on day 11) \\
\hline \multirow[t]{8}{*}{ Exp. $2^{B}$} & $+/+$ & M & Yes (day 13) \\
\hline & $+/+$ & M & No \\
\hline & $+/-$ & $\mathrm{F}$ & Yes (day 13) \\
\hline & $+/-$ & $\mathrm{F}$ & Yes (day 25) \\
\hline & $+/-$ & M & Yes (day 20) \\
\hline & $-1-$ & $\mathrm{F}$ & ND (died on day 20) \\
\hline & $-1-$ & $\mathrm{F}$ & ND (died on day 20) \\
\hline & $-/-$ & M & ND (sacrificed on day 20 ) \\
\hline \multirow[t]{15}{*}{ Exp. $3^{c}$} & $+/+$ & $\mathrm{F}$ & Yes (day 11) \\
\hline & $+/+$ & $\mathrm{F}$ & Yes (day 22) \\
\hline & $+/+$ & M & Yes (day 27) \\
\hline & $+/+$ & M & No \\
\hline & $+/+$ & M & No \\
\hline & $+/-$ & $\mathrm{F}$ & Yes (day 14) \\
\hline & $+/-$ & $\mathrm{F}$ & Yes (day 22) \\
\hline & $+/-$ & $\mathrm{F}$ & Yes (day 22) \\
\hline & $+/-$ & $\mathrm{F}$ & Yes (day 22) \\
\hline & $+/-$ & M & No \\
\hline & $-/-$ & $\mathrm{F}$ & ND (died on day 7) \\
\hline & $-/-$ & $\mathrm{F}$ & ND (died on day 22) \\
\hline & $-/-$ & $\mathrm{F}$ & ND (died on day 22) \\
\hline & $-1-$ & M & ND (died on day 7) \\
\hline & $-1-$ & M & ND (died on day 22) \\
\hline
\end{tabular}

CY dissolved in PBS was injected i.p. (0.2 mg/g body weight) into mice on day 0 and day 14 . Blood glucose levels were measured every day, and mice were diagnosed as diabetic when blood glucose levels exceeded $250 \mathrm{mg} / \mathrm{dl}$. Observation was terminated 28 days after the first $\mathrm{CY}$ injection. ANine-week-old mice backcrossed onto NOD mice for 6 generations were used for the experiment. BSeven-week-old mice backcrossed onto NOD mice for 6 generations were used for the experiment. CNine-week-old mice backcrossed onto NOD mice for 8 and 9 generations were used for the experiment. Exp., experiment; ND, not determined (reason described in parentheses). 


\section{Table 3}

Development of diabetes in NOD-scid mice transferred with mature T cells

\begin{tabular}{|c|c|c|c|c|c|c|}
\hline & \multirow[t]{2}{*}{ Donor } & \multirow[t]{2}{*}{ Diabetes development } & \multirow[t]{2}{*}{ BW (g) } & \multicolumn{3}{|c|}{ Histology of pancreas } \\
\hline & & & & $\begin{array}{l}\text { Peri-insular } \\
\text { Iymphocytic } \\
\text { infiltration }\end{array}$ & $\begin{array}{l}\text { Loss of } \\
\beta \text { cell } \\
\text { islets }\end{array}$ & $\begin{array}{l}\text { Atrophy } \\
\text { of acinar } \\
\text { tissues }\end{array}$ \\
\hline \multirow[t]{10}{*}{ Exp. $1^{A}$} & $+/+$ & ND (sacrificed on day 51) & 19.3 & ++ & - & - \\
\hline & t/+ & Yes (day 77) & 22.1 & ++ & + & - \\
\hline & t/+ & Yes (day 120) & 24.6 & ++ & ++ & - \\
\hline & $+/+$ & Yes (day 140) & 23.3 & + & ++ & - \\
\hline & t/+ & No & 24.4 & ++ & - & - \\
\hline & $-1-$ & ND (sacrificed on day 51) & 12.4 & ++ & + & + \\
\hline & $-1-$ & ND (died on day 57) & ND (not available) & ND (not available) & ND (not available) & ND (not available) \\
\hline & $-1-$ & No & 18.4 & ++ & - & ++ \\
\hline & $-1-$ & No & 17.2 & ++ & - & ++ \\
\hline & $-1-$ & No & 17.6 & ++ & - & ++ \\
\hline \multirow[t]{5}{*}{ Exp. $2^{B}$} & $+/+$ & Yes (day 77) & 21.6 & + & ++ & - \\
\hline & $+/+$ & ND (sacrificed on day 95) & 20.8 & ++ & ++ & - \\
\hline & $-1-$ & ND (sacrificed on day 90) & 15.4 & ++ & - & + \\
\hline & $-1-$ & ND (sacrificed on day 95) & 17.2 & ++ & - & + \\
\hline & $-/-$ & ND (sacrificed on day 95) & 16.2 & ++ & + & + \\
\hline
\end{tabular}

Thy $1{ }^{+}$cells purified from 7-week-old female mouse spleens of either 3 control mice or 3 Aire-deficient NOD mice were injected i.v. into 8-week-old female NOD-scid mice ( $4 \times 10^{6}$ cells per mouse), and development of diabetes was monitored for 20 weeks. Blood glucose levels were measured every week, and mice were diagnosed as diabetic when blood glucose levels exceeded $250 \mathrm{mg} / \mathrm{dl}$. AMice backcrossed onto NOD mice for 6 generations were used. ${ }^{\mathrm{A}}$ Mice backcrossed onto NOD mice for 8 generations were used. BW, body weight. ++, severe; +, moderate; -, not remarkable.

NOD mouse T cells was sacrificed at day 51 for histological comparison with a NOD-scid mouse transferred with Aire-deficient NOD mouse T cells. In contrast, none of the NOD-scid mice transferred with Aire-deficient NOD mouse T cells developed diabetes; we had to sacrifice 1 mouse at day 51 because of severe sickness, and another mouse died during the observation period (Table 3, experiment 1). All the mice transferred with Aire-deficient NOD mouse $\mathrm{T}$ cells showed body-weight loss before sacrifice. It is important to mention that we used splenic $T$ cells isolated from Aire-deficient NOD mice before their body-weight loss had begun (i.e., 7 weeks old). Furthermore, flow-cytometric analysis of thymocytes and splenocytes isolated from the Aire-deficient NOD mouse donors used in this experiment showed no obvious changes compared with those from control mice (data not shown).

Histological evaluation showed that the NOD-scid mice transferred with Aire-deficient NOD mouse T cells recapitulated the pathological changes seen in untreated Aire-deficient NOD mice; acinar tissues were severely destroyed by the infiltrating lymphoid cells, and only pancreatic ducts and relatively well-preserved $\beta$ cell islets remained (Figure $2 \mathrm{C}$ and Table 3 ). In contrast, reduced size and numbers of $\beta$ cell islets with lymphoid cell infiltration were the predominant pathological features of NOD-scid mice transferred with control mouse T cells, as seen in untreated Aire-sufficient NOD mice (Figure 2, A and C). These results indicate that Aire-deficient NOD mice are resistant to the development of diabetes as a result of alteration of intra-pancreatic target-organ specificity from $\beta$ cell islets toward acinar cells. A second set of experiments with more backcrossed mice showed similar results (Table 3, experiment 2).

Polyclonal B cell activation in Aire-deficient NOD mice. Serum IgM and all subclasses of IgG except for IgG3 were significantly elevated in Aire-deficient NOD mice (Figure 3C). These increased serum Ig levels, together with augmented autoimmunity in Aire-deficient NOD mice, as described above, were associated with the produc- tion of autoantibodies against various organs. When serum from Aire-deficient NOD mice was tested for reactivity against pancreas, stomach, kidney, and liver, all sera contained autoantibodies of the IgG class against the broad spectrum of organs, as detected with immunofluorescence (Supplemental Figure 3 and Supplemental Table 3). Interestingly, autoantibodies from Aire-deficient NOD mice reacted with acinar cells more strongly than with $\beta$ cell islets in the pancreas (Supplemental Figure 3, top right panel), which is consistent with the predominant autoimmune attack against acinar cells. In contrast, no such activities were observed in sera from control littermates. These results suggest induction of polyclonal B cell activation in Aire-deficient NOD mice, which has not been observed either in Aire-deficient mice with non-NOD backgrounds $(13,14)$ or in the original NOD mice. This was unexpected, because both Aire-deficient mice and NOD mice are considered to be models of $\mathrm{T}$ cell-mediated organ-specific autoimmunity rather than systemic autoimmunity. Serum cytokine levels, including those of IFN- $\alpha$, IL-2, IL-4, and IL-6, in Aire-deficient NOD mice were below the limit of detection, as with control littermates. Expression of $\mathrm{B}$ cell surface markers, such as CD40, CD80, CD86, and B7-related protein 1 (B7RP-1), on splenic B cells was also unchanged in Airedeficient NOD mice (S. Niki et al., unpublished data).

Identification of PDIP as an autoantigen recognized by Aire-deficient NOD mouse serum. Preferential recognition of acinar cells by Aire-deficient NOD mouse serum with immunofluorescence prompted us to identify target antigen(s) that might be associated with an alteration of intra-pancreatic target-organ specificity. We first characterized target antigen(s) by Western blot analysis using proteins extracted from the pancreas as well as other tissues (Figure 4A). Protein extracted from the pancreas showed a discrete band of approximately $65 \mathrm{kDa}$, and this was observed in all the Aire-deficient NOD mouse sera tested (10 of 10). A band of the same size was also observed from the protein extracted from the stomach. After $N$-glycosidase F treatment, 
A

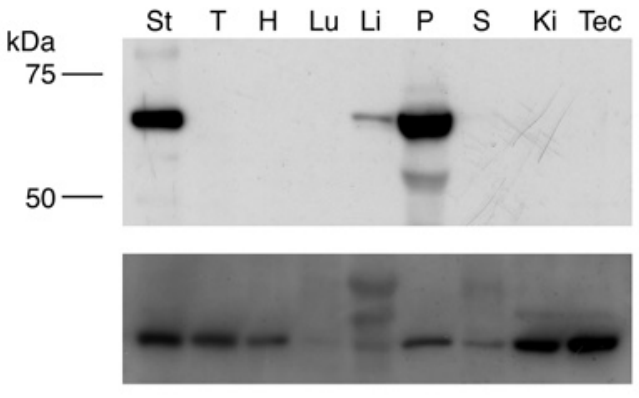

IB: anti-actin

\section{IB: Aire KO serum}

B

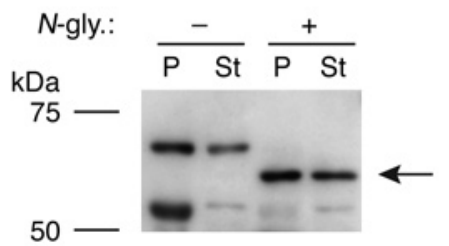

C

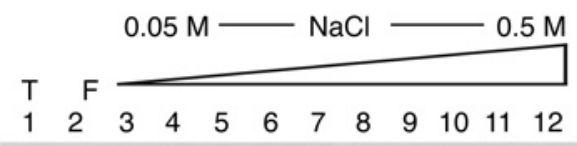

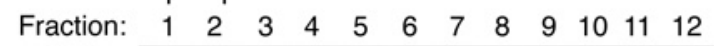
$\mathrm{kDa}$
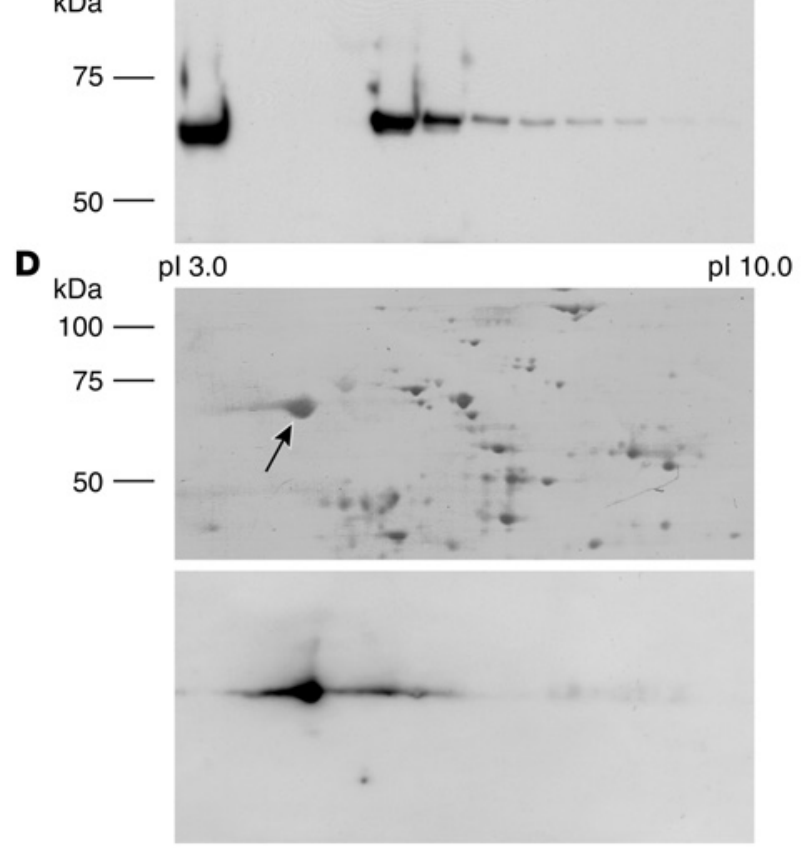

\section{Figure 4}

Identification of PDIp as an autoantigen recognized by Aire-deficient NOD mouse serum. (A) Detection of autoantigen from the stomach and pancreas with Western blot analysis using Aire-deficient NOD mouse serum. The same blot was probed with anti-actin Ab (bottom panel). St, stomach; T, thymus; H, heart; Lu, lung; Li, liver; P, pancreas; S, spleen; Ki, kidney; Tec, mouse thymic epithelial cell line. (B) The target antigen is present as a glycoprotein in both the pancreas and the stomach. After $\mathrm{N}$-glycosidase $\mathrm{F}$ treatment (marked as " +"), both bands similarly migrated to lower positions (indicated by an arrow). Western blot analysis with Aire-deficient NOD mouse serum was used for the detection. P, pancreas; St, stomach. (C) Purification of autoantigen by anion-exchange chromatography. Each fraction eluted with increasing concentrations of $\mathrm{NaCl}$ was tested for the presence of autoantigen by Western blot analysis. T, total cell lysate; F, flow-through fraction. (D) Identification of autoantigen by 2-dimensional gel electrophoresis. Proteins from fraction 5 shown in $\mathbf{C}$ were subjected to 2-dimensional gel electrophoresis (top panel). Western blot analysis identified a discrete signal (bottom panel), and the corresponding spot in the gel was subjected to mass spectrometry (indicated by an arrow in the top panel). pl, isoelectric point.

both bands similarly migrated to lower positions, suggesting that the target antigen is a glycoprotein present in both the pancreas and the stomach (Figure 4B). Because epithelial cells from the stomach were easier to obtain and handle, we attempted to isolate the target antigen from these cells. After purification by anion-exchange chromatography, the target antigen was found to be present most abundantly in the fraction eluted with $0.15 \mathrm{M} \mathrm{NaCl}$ (Figure 4C, fraction 5), and therefore this fraction was further subjected to 2dimensional gel electrophoresis (Figure 4D, top panel). Western blot analysis of the 2-dimensional gel revealed a discrete spot (Figure 4D, bottom panel), which was identified as PDIp by mass spectrometry. The change in the molecular weight of the target antigen after $N$-glycosidase F treatment (Figure 4B) was consistent with the characteristics of PDIp $(20,21)$. Furthermore, Aire-deficient NOD mouse serum, but not control mouse serum, showed reactivity against bacterially expressed glutathione-S-transferase-PDIp fusion protein, but not against glutathione-S-transferase alone (Supplemental Figure 4). Although PDIp was originally reported to be pancreas-specific in humans, we detected PDIp expression in the mouse stomach by Northern blot analysis (data not shown) and in fact identified PDIp as an autoantigen using proteins extracted from the stomach, as described above. More importantly, it has been reported that PDIp is restricted to acinar cells in the pancreas, at least in humans $(20,21)$. Despite the development of autoimmunity against PDIp, transcriptional expression of PDIP was retained in TECs (Table 1) or total thymus (Supplemental Table 2) of Aire-deficient NOD mice.

\section{Discussion}

In order to gain further insight into the roles of AIRE in the establishment of central tolerance, we have established NOD mice lacking Aire. We specifically chose NOD mice because of their well-established intrinsic defect in thymocyte apoptosis during negative selection (6-8), hoping that the role of Aire as a stromal factor in central tolerance might be better investigated by combination with a defective reciprocal factor (i.e., pathogenic NOD thymocytes). The results highlighted unique features of Aire that control target-organ specificity. Although there are many examples of gene-targeted mice in which distinct target organs are involved in autoimmune destruction depending on the genetic background of the animal, as we have also reported for Aire-deficient mice (14), the results presented in this study are striking in that deletion of a single gene, Aire, altered a discrete target-organ specificity of NOD mice on a fixed genetic background. 
Aire in TECs has been suggested to regulate promiscuous gene expression, thereby controlling autoimmunity (13). In fact, TECs isolated from Aire-deficient NOD mice showed reduced expression of several tissue-specific genes, including insulin, as observed in non-autoimmune-prone mouse backgrounds $(13,14,16)$. Given that insulin is a prime target antigen recognized by autoreactive $T$ cells in NOD mice (19), the finding that $\beta$ cell islets were relatively less affected by autoimmune attack in Aire-deficient NOD mice, despite repressed insulin expression in their TECs (Table 1), does not support the concept that Aire-dependent transcriptional control of tissue-specific antigen genes in TECs accounts for Aire-dependent autoimmunity. Furthermore, thymic expression of PDIP, a tissuespecific antigen recognized by Aire-deficient NOD mouse serum, was not downregulated, as demonstrated in this study (Table 1 and Supplemental Table 2). Thus, there is still a lack of experimental evidence to connect the postulated roles of Aire in the transcriptional regulation of tissue-specific antigen expression with efficient elimination of autoreactive T cells. We currently favor the idea that Aire may regulate the processing and/or presentation of self-proteins in order for autoreactive $T$ cells to be appropriately eliminated within the thymus $(14,18)$. In this regard, it is also noteworthy that bone marrow-derived cells, most likely DCs, in the thymus can acquire self-antigens presented by medullary TECs and cross-present them to developing thymocytes to enforce self-tolerance (27). It is therefore possible that loss of AIRE function in medullary TECs may result in insufficient uptake of self-proteins from medullary TECs by DCs necessary for cross-presentation.

Given that Tregs arise from relatively high-avidity interactions with self-peptide-MHC complexes just below the threshold for negative selection (28-30), it was somewhat unexpected that Aire deficiency had no major impact on the production as well as the suppressive function of Tregs in non-autoimmune-prone mouse backgrounds $(14,18)$. Similarly, we have demonstrated that the numbers of Foxp $3^{+}$Tregs were not changed in Aire-deficient mice even on an autoimmune-prone NOD mouse background (Figure 3A). This lack of a major impact of Aire on the production and/or function of Tregs appears to be supported by a recent report describing the phenotypes of NOD mice in which Tregs were depleted by in vivo IL-2 neutralization (31); the effect of in vivo IL-2 neutralization has mainly been attributed to the loss of homeostatic maintenance of Tregs. In contrast to the Aire-deficient NOD mice described in the present study, NOD mice depleted of Tregs showed early onset of diabetes (31). Furthermore, NOD mice depleted of Tregs demonstrated target-organ specificity, such as neuritis, different from that seen in Aire-deficient NOD mice. Thus, although it remains possible that in vivo IL-2 neutralization does more than deplete Tregs, the phenotypes of Aire-deficient NOD mice are different from those seen in NOD mice deficient in Tregs, suggesting that Aire does not play major roles in the regulation of Treg-mediated autoimmunity.

Numerous factors have been reported to modulate the diabetic process in NOD mice, many of which affect $\mathrm{T}$ cell function and/ or communication between APCs and T cells, both mainly in the periphery $(3,32)$. Among these, one recent report merits attention (33). NOD mice deficient in programmed cell death 1 (PD-1), an immunoinhibitory receptor belonging to the CD28/CTL-associated antigen- 4 family, exhibited accelerated and augmented insulitis compared with PD-1-sufficient NOD mice. Sialoadenitis was also accelerated in PD-1-deficient NOD mice. Of importance, other organs have never been reported to be affected by the absence of PD-1 despite the severe lesions of pancreas and salivary glands, suggest- ing that target-organ specificity was maintained in NOD mice deficient in PD-1. In contrast, alteration of intra-pancreatic target-organ specificity together with an expansion of target-organ spectrum was observed in Aire-deficient NOD mice. The different effects of abrogation of PD-1 or Aire underscore the existence of autoimmune modulators with unique actions. PD-1 may be a general regulator of the autoimmune process without governing target-organ specificity, at least on the NOD background (33), whereas Aire additionally controls target-organ specificity on the same background.

We have identified PDIp as an autoantigen recognized by serum from Aire-deficient NOD mice. The pathogenic roles of anti-PDIp autoantibody in the alteration of intra-pancreatic target-organ specificity remain to be studied. Because NOD-scid mice inoculated with Aire-deficient NOD mouse T cells alone developed pancreatic lesions quite similar to those of untreated Aire-deficient NOD mice, production of anti-PDIp autoantibody may not be relevant to the disease process but could be secondary to the massive destruction of acinar cells. Nevertheless, as Aire-deficient NOD mice developed autoimmunity against PDIp, and PDIp expression is restricted to acinar cells $(20,21)$, Aire clearly plays a significant role in the alteration of intra-pancreatic target-organ specificity demonstrated in this study. Furthermore, identification of this tissue-specific antigen as an autoantigen recognized by Aire-deficient NOD mice helped us to strengthen our current model of Aire function described above, because $\alpha$-fodrin, which we initially identified as an autoantigen that was not downregulated in Aire-deficient thymus, is a ubiquitous, but not tissue-specific, antigen (14).

It remains unexplained why the abrogation of Aire resulted in the alteration of intra-pancreatic target-organ specificity from $\beta$ cell islets to acinar cells. Even though abrogation of Aire could additionally impair the presentation of pancreatic acinar-cell antigen(s), thereby provoking autoimmunity against these cells by a mechanism such as proposed above, autoreactivity against $\beta$ cell islets (or possibly insulin) should still remain, and we would expect to see the development of diabetes in Aire-deficient NOD mice with at least a similar frequency to that in Aire-sufficient NOD mice. However, Aire-deficient NOD mice demonstrated resistance to the development of diabetes, both in their natural history and in 2 models of induced diabetes (CY injection and T cell transfer into NOD-scid mice). One possible explanation for this alteration of intra-pancreatic target-organ specificity is that establishment of self-tolerance for $\beta$-cell-islet antigen(s) and establishment of self-tolerance for acinarcell antigen(s) are not independent processes in the negative selection niche in the thymus; impaired negative selection against acinarcell antigen(s) by loss of Aire could, by an unknown mechanism, have relieved abnormal negative selection against $\beta$ cell antigen(s). Alternatively, the capacity for autoimmune attack against $\beta$ cell islets might have been exhausted by mobilization of immune cells toward the destruction of a better target in the form of neighboring acinar cells. The reasoning behind both these explanations may be much too simplistic, and better understanding of the nature of target-organ specificity as well as of the primary function of AIRE requires new experimental systems to approach these issues.

The finding that abrogation of Aire in NOD mice, even though it was achieved by a mechanism involving alteration of intra-pancreatic target-organ specificity, did not accelerate the development of diabetes is obviously inconsistent with the extremely early onset of diabetes in Aire-deficient double-transgenic mice with model antigens expressed in the thymus together with TCRs recognizing the corresponding antigens $(18,34)$. However, this discrepancy between 
the present study and the transgenic studies does not argue against an essential role of Aire in the prevention of autoimmune disease. Rather, we think that the difference is simply due to the uniqueness of each model, in which Aire might exert different roles in the disease process. The function of Aire in controlling target-organ specificity was recognizable only in the present study, in which clonal diversification of thymocytes was possible during the course of negative selection. In contrast, prevention of an autoimmune process of fixed target-organ specificity (i.e., progression from innocuous insulitis to overt diabetes) was the predominant feature of Aire observed in the transgenic studies because of the predefined TCR specificities in which clonal diversification was not available $(18,34)$.

Because both Aire-deficient and NOD mice demonstrate organspecific autoimmune pathology rather than a systemic autoimmunity such as SLE, the polyclonal B cell activation in Aire-deficient NOD mice was unexpected. Local Ig production by the CD $138^{+}$cell clusters in the pancreas might be responsible for the elevation of serum Ig levels (Supplemental Figure 2B). In this respect, it is possible to speculate that an Aire-deficient thymic microenvironment will produce more $T$ cells of a certain subset(s) that promotes B cell maturation into plasma cells, in a manner similar to that in which follicular Th cells help to produce high titers of autoantibodies consistent with polyclonal B cell activation (35). In fact, flow-cytometric analyses of splenic T cells from Aire-deficient NOD mice have demonstrated increased percentages of the $\mathrm{CD}_{4} 4^{\text {high }} \mathrm{ICOS}^{+}$ population (Figure 3B). Interestingly, a gene-chip analysis of genes whose expression levels differed between Aire-deficient and control medullary TECs identified several genes that might affect $T$ cell function and/or differentiation into distinct $\mathrm{T}$ cell subtypes, including genes involved in antigen processing and/or presentation and genes coding for chemokines (18).

Finally, a single report has suggested altered functions of Aire and/or Aire ${ }^{+}$cells that could be relevant to the autoimmune phenotypes seen in NOD mice (36). Although NOD thymi have been shown by Western blot analysis to express Aire protein at normal levels, and the size and shape of the Aire nuclear dots from NOD thymus resembled the pattern seen in control thymus, as we have also observed (Supplemental Figure 1B), it was reported that Aire ${ }^{+}$ cells from NOD mice contained large intranuclear vacuoles that seemed to displace the Aire protein to the periphery of the nuclei, suggesting that abnormal functions of Aire and/or Aire ${ }^{+}$cells in TECs may contribute to the development of the autoimmune phenotypes of NOD mice (36). Our present study, however, clearly demonstrated that abrogation of Aire in NOD mice modulated the autoimmune phenotypes of NOD mice, suggesting that Aire and/or Aire ${ }^{+}$cells in NOD mice are functionally competent.

In conclusion, our present study has provided unique insights into the roles of Aire in the pathogenesis of organ-specific autoimmune disease through combination with the autoimmune-prone NOD mouse model. In turn, we have obtained novel perspectives on the autoimmune process in NOD mice through loss of the fundamental stromal function of Aire. More generally, we believe that understanding the relationship between AIRE gene malfunction and the breakdown of self-tolerance promises to help unravel the pathogenesis not only of APECED but also of other types of autoimmune disease.

\section{Methods}

Mice. NOD/Shi Jic mice and NOD/Shi-scid Jic mice were purchased from CLEA Japan Inc., and Rag2-deficient mice on a BALB/c background were from Taconic. Aire-deficient mice of mixed background $\left(\mathrm{H}-2^{\mathrm{b} / \mathrm{k}} \times \mathrm{H}-2^{\mathrm{b}}\right)$ were generated as previously reported (14). The mice were maintained under pathogen-free conditions and were handled in accordance with the Guidelines for Animal Experimentation of University of Tokushima School of Medicine. All animal experiments were approved by the Animal Experimentation Committee of Tokushima University.

Pathology. Formalin-fixed tissue sections were subjected to H\&E staining, and 2 pathologists independently evaluated the histology without being informed of the detailed condition of the individual mouse.

Flow-cytometric analysis. Thymus and spleen cell suspensions were stained with the following mAbs: anti-CD45R/B220, anti-CD3, anti-CD4, anti$\mathrm{CD} 8$, anti-CD44, and anti-Foxp3 (eBioscience). Staining for Foxp3-expressing cells was performed according to the manufacturer's instructions.

Immunohistochemistry. Immunohistochemical analysis of the thymus with ER-TR5 (37) and UEA-1-FITC (Vector Laboratories) was performed as previously described (23). Polyclonal anti-Aire Ab was produced by immunization of rabbits with peptides corresponding to the $\mathrm{COOH}$-terminal portion of mouse Aire, and Alexa 488-conjugated donkey anti-rabbit IgG (Invitrogen Corp.) was used as a secondary Ab for detection. For the detection of autoantibodies, mouse serum was incubated with various organs obtained from Rag2-deficient mice. FITC-conjugated anti-mouse IgG Ab (Southern Biotechnology Associates Inc.) was used for detection (23).

TEC preparation. TECs were prepared as described previously (14). Briefly, thymic lobes were isolated from 2 mice for each group and cut into small pieces. The fragments were gently rotated in RPMI 1640 medium (Invitrogen Corp.) supplemented with 10\% heat-inactivated FCS (Invitrogen Corp.), $20 \mathrm{mM}$ HEPES, $100 \mathrm{U} / \mathrm{ml}$ penicillin, $100 \mu \mathrm{g} / \mathrm{ml}$ streptomycin, and $50 \mu \mathrm{M} 2$-mercaptoethanol at $4{ }^{\circ} \mathrm{C}$ for 30 minutes, and dispersed further with pipetting to remove the majority of thymocytes. The resulting thymic fragments were digested with $0.125 \%$ collagenase D (Roche Molecular Biochemicals) and $10 \mathrm{U} / \mathrm{ml}$ DNase I (Roche Molecular Biochemicals) in RPMI 1640 at $37^{\circ} \mathrm{C}$ for 15 minutes. The supernatants, containing dissociated TECs, were saved, and the remaining thymic fragments were further digested with collagenase $\mathrm{D}$ and DNase I. This step was repeated twice, and the remaining thymic fragments were digested with $0.125 \%$ collagenase/dispase (Roche Applied Science) and DNase I at $37^{\circ} \mathrm{C}$ for 30 minutes. The supernatants from this digest were combined with the supernatants from the collagenase digests, and the mixture was centrifuged for 5 minutes at $450 \mathrm{~g}$. The cells were suspended in PBS containing 5 mM EDTA and $0.5 \%$ FCS and kept on ice for 10 minutes. CD45- thymic stromal cells were then purified by depletion of $\mathrm{CD} 45^{+}$cells with MACS CD45 MicroBeads (Miltenyi Biotec) according to the manufacturer's instructions. The resulting preparations contained approximately $60 \%$ epithelial cell adhesion molecule-positive cells and less than $10 \%$ thymocytes (i.e., CD4/CD8 single-positive and CD4/CD8 doublepositive cells) as determined by flow-cytometric analysis.

Real-time PCR. RNA was extracted from TECs with the High Pure RNA Isolation Kit (Roche Applied Science) and made into cDNA with the cDNA Cycle Kit (Invitrogen Corp.) according to the manufacturer's instructions. Real-time PCR for quantification of tissue-specific antigen genes was carried out as previously described (14). The primers and the probe for PDIP were as follows: primers, $5^{\prime}$-AGGCCAAAATAATTCAGCACATG-3' and 5'-TTTGTGAACCAGACACTAGCTCAAC-3'; probe, 5'-FAM-TCCACCATTACGAACAACACCTGCCC-3'.

CY-induced diabetes. CY (Sigma-Aldrich) dissolved in PBS was injected i.p. $(0.2 \mathrm{mg} / \mathrm{g}$ body weight $)$ into mice on day 0 and day 14 . Mice were diagnosed as diabetic when blood glucose levels exceeded $250 \mathrm{mg} / \mathrm{dl}$. Observation was terminated 28 days after the first CY injection.

Transfer of peripheral T cells into NOD-scid mice. Thy $1^{+}$cells were isolated from the spleen, as previously described (38). In brief, the spleen cell suspensions were depleted of rbcs by osmotic lysis, and their Thy $1^{+}$cells were purified with CD90 (Thy1.2) MicroBeads (Miltenyi Biotec). The resulting preparations con- 
tained approximately $95 \%$ Thy $1^{+}$cells. The purified Thy $1^{+}$cells were injected i.v. $\left(4 \times 10^{6}\right.$ cells per mouse), and development of diabetes was monitored for 20 weeks. Diagnosis of diabetes was performed as described above.

Measurement of serum Ig levels. The concentrations of Ig in the sera were measured by a sandwich ELISA using a clonotyping system with HRP (Southern Biotechnology Associates Inc.), as previously described (38).

Identification of autoantigen. Gastric epithelial cells were recovered by scratching of the surface of the glandular stomach from $\mathrm{BALB} / \mathrm{c}$ mice with microscope slides. The cells were lysed in buffer containing $1 \%$ NP-40, $10 \mathrm{mM}$ Tris$\mathrm{HCl}$ ( $\mathrm{pH}$ 7.4), $0.4 \mathrm{mM}$ EDTA, $0.15 \mathrm{M} \mathrm{NaCl}, 10 \mu \mathrm{g} / \mathrm{ml}$ aprotinin, $10 \mu \mathrm{g} / \mathrm{ml}$ leupeptin, and $1 \mathrm{mM}$ phenylmethylsulfonyl fluoride, and the mixture was then centrifuged for 10 minutes at 20,000 $\mathrm{g}$. The supernatant was applied to DE52 resin (Whatman) that had been equilibrated with buffer containing $50 \mathrm{mM}$ Tris- $\mathrm{HCl}(\mathrm{pH} 8.0)$, hereafter referred to as buffer A. Proteins were eluted from the column stepwise with buffer A supplemented with 0.05-0.5 M $\mathrm{NaCl}$. Fractions containing autoantigen were determined by Western blot analysis. One milliliter of the fraction containing autoantigen (i.e., the fraction eluted with $0.15 \mathrm{M} \mathrm{NaCl}$ ) was concentrated and then applied to an Immobiline DryStrip (GE Healthcare) (pI 3-10) using Ettan IPGphor II (GE Healthcare). After isoelectric focusing electrophoresis, proteins were separated by SDS-PAGE on a $9 \%$ gel and stained with Coomassie brilliant blue. The stained spot corresponding to the autoantigen determined by Western blot analysis was excised from the gel. The protein was subjected to in-gel reduction, $S$-carboxyamidomethylation, and digestion with trypsin. The resulting peptides were analyzed by LCQ ion-trap mass spectrometry (Thermo Electron Corp.) (39). $N$-glycosidase F treatment (Roche Molecular Biochemicals) was then carried out at $37^{\circ} \mathrm{C}$ for 24 hours.

Statistics. Statistics were analyzed with 2 -tailed Student's $t$ test; $P$ values less than 0.05 were considered significant.

\section{Acknowledgments}

We thank W. van Ewijk and M. Itoi for mAb ER-TR5. We also thank S. Hori for valuable suggestions. This work was supported in part by Special Coordination Funds of the Ministry of Education, Culture, Sports, Science and Technology (MEXT), and by a Grant-in-Aid for Scientific Research from the MEXT (17047028 and 17390291 to M. Matsumoto).

Received for publication September 26, 2005, and accepted in revised form February 28, 2006.

Address correspondence to: Mitsuru Matsumoto, Division of Molecular Immunology, Institute for Enzyme Research, University of Tokushima, 3-18-15 Kuramoto, Tokushima 770-8503, Japan. Phone: 81-88-633-7432; Fax: 81-88-633-7434; E-mail: mitsuru@ ier.tokushima-u.ac.jp.

\section{S. Niki and K. Oshikawa contributed equally to this work.}

1. Eisenbarth, G.S. 1986. Type I diabetes mellitus. A chronic autoimmune disease. N. Engl. J. Med. 314:1360-1368.

2. Tisch, R., and McDevitt, H. 1996. Insulin-dependent diabetes mellitus. Cell. 85:291-297.

3. Atkinson, M.A., and Leiter, E.H. 1999. The NOD mouse model of type 1 diabetes: as good as it gets? Nat. Med. 5:601-604.

4. Anderson, M.S., and Bluestone, J.A. 2005. The NOD mouse: a model of immune dysregulation. Annu. Rev. Immunol. 23:447-485.

5. Todd, J.A., and Wicker, L.S. 2001. Genetic protection from the inflammatory disease type 1 diabetes in humans and animal models. Immunity. 15:387-395.

6. Kishimoto, H., and Sprent, J. 2001. A defect in central tolerance in NOD mice. Nat. Immunol. 2:1025-1031.

7. Zucchelli, S., et al. 2005. Defective central tolerance induction in NOD mice: genomics and genetics. Immunity. 22:385-396.

8. Liston, A., et al. 2004. Generalized resistance to thymic deletion in the NOD mouse: a polygenic trait characterized by defective induction of Bim. Immunity. 21:817-830.

9. von Boehmer, H., et al. 2003. Thymic selection revisited: how essential is it? Immunol. Rev. 191:62-78

10. Björses, P., Aaltonen, J., Horelli-Kuitunen, N., Yaspo, M.L., and Peltonen, L. 1998. Gene defect behind APECED: a new clue to autoimmunity. Hum. Mol. Genet. 7:1547-1553.

11. Pitkänen, J., and Peterson, P. 2003. Autoimmune regulator: from loss of function to autoimmunity. Genes Immun. 4:12-21.

12. Ramsey, C., et al. 2002. Aire deficient mice develop multiple features of APECED phenotype and show altered immune response. Hum. Mol. Genet. 11:397-409.

13. Anderson, M.S., et al. 2002. Projection of an immunological self-shadow within the thymus by the Aire protein. Science. 298:1395-1401.

14. Kuroda, N., et al. 2005. Development of autoimmunity against transcriptionally unrepressed target antigen in the thymus of Aire-deficient mice. J. Immunol. 174:1862-1870.

15. Kyewski, B., and Derbinski, J. 2004. Self-represen- tation in the thymus: an extended view. Nat. Rev. Immunol. 4:688-698.

16. Derbinski, J., et al. 2005. Promiscuous gene expression in thymic epithelial cells is regulated at multiple levels. J. Exp. Med. 202:33-45.

17. Liston, A., Lesage, S., Wilson, J., Peltonen, L., and Goodnow, C.C. 2003. Aire regulates negative selection of organ-specific T cells. Nat. Immunol. 4:350-354

18. Anderson, M.S., et al. 2005. The cellular mechanism of Aire control of $\mathrm{T}$ cell tolerance. Immunity. 23:227-239.

19. Nakayama, M., et al. 2005. Prime role for an insulin epitope in the development of type 1 diabetes in NOD mice. Nature. 435:220-223.

20. Desilva, M.G., et al. 1996. Characterization and chromosomal localization of a new protein disulfide isomerase, PDIp, highly expressed in human pancreas. DNA Cell Biol. 15:9-16.

21. Desilva, M.G., Notkins, A.L., and Lan, M.S. 1997. Molecular characterization of a pancreas-specific protein disulfide isomerase, PDIp. DNA Cell Biol. 16:269-274.

22. Jiang, W., Anderson, M.S., Bronson, R., Mathis, D., and Benoist, C. 2005. Modifier loci condition autoimmunity provoked by Aire deficiency. J. Exp. Med. 202:805-815.

23. Kajiura, F., et al. 2004. NF-кB-inducing kinase establishes self-tolerance in a thymic stromadependent manner. J. Immunol. 172:2067-2075.

24. Harada, M., and Makino, S. 1984. Promotion of spontaneous diabetes in non-obese diabetesprone mice by cyclophosphamide. Diabetologia. 27:604-606.

25. Yasunami, R., and Bach, J.F. 1988. Anti-suppressor effect of cyclophosphamide on the development of spontaneous diabetes in NOD mice. Eur. J. Immunol. 18:481-484.

26. Christianson, S.W., Shultz, L.D., and Leiter, E.H. 1993. Adoptive transfer of diabetes into immunodeficient NOD-scid/scid mice. Relative contributions of $\mathrm{CD}^{+}$and $\mathrm{CD}^{+} \mathrm{T}$-cells from diabetic versus prediabetic NOD.NON-Thy-1a donors. Diabetes. 42:44-55.

27. Gallegos, A.M., and Bevan, M.J. 2004. Central tolerance to tissue-specific antigens mediated by direct and indirect antigen presentation. J. Exp. Med. 200:1039-1049.

28. Jordan, M.S., et al. 2001. Thymic selection of $\mathrm{CD} 4{ }^{+} \mathrm{CD} 25^{+}$regulatory $\mathrm{T}$ cells induced by an agonist self-peptide. Nat. Immunol. 2:301-306.

29. Apostolou, I., Sarukhan, A., Klein, L., and von Boehmer, H. 2002. Origin of regulatory T cells with known specificity for antigen. Nat. Immunol. 3:756-763.

30. Sakaguchi, S. 2004. Naturally arising $\mathrm{CD}^{+}$regulatory $\mathrm{T}$ cells for immunologic self-tolerance and negative control of immune responses. Annu. Rev. Immunol. 22:531-562.

31. Setoguchi, R., Hori, S., Takahashi, T., and Sakaguchi, S. 2005. Homeostatic maintenance of natural Foxp $3^{+} \mathrm{CD} 25^{+} \mathrm{CD}^{+}$regulatory $\mathrm{T}$ cells by interleukin (IL)-2 and induction of autoimmune disease by IL-2 neutralization. J. Exp. Med. 201:723-735.

32. Shoda, L.K., et al. 2005. A comprehensive review of interventions in the NOD mouse and implications for translation. Immunity. 23:115-126.

33. Wang, J., et al. 2005. Establishment of NOD-Pdcd1 $1^{-/}$ mice as an efficient animal model of type I diabetes. Proc. Natl. Acad. Sci. U. S. A. 102:11823-11828.

34. Liston, A., et al. 2004. Gene dosage-limiting role of Aire in thymic expression, clonal deletion, and organ-specific autoimmunity. J. Exp. Med. 200:1015-1026.

35. Vinuesa, C.G., et al. 2005. A RING-type ubiquitin ligase family member required to repress follicular helper T cells and autoimmunity. Nature. 435:452-458.

36. Heino, M., et al. 2000. RNA and protein expression of the murine autoimmune regulator gene (Aire) in normal, RelB-deficient and in NOD mouse. Eur. J. Immunol. 30:1884-1893.

37. van Vliet, E., Melis, M., and van Ewijk, W. 1984. Monoclonal antibodies to stromal cell types of the mouse thymus. Eur. J. Immunol. 14:524-529.

38. Yamada, T., et al. 2000. Abnormal immune function of hemopoietic cells from alymphoplasia (aly) mice, a natural strain with mutant NF- $\mathrm{KB}$-inducing kinase. J. Immunol. 165:804-812.

39. Matsumoto, M., et al. 2005. Large-scale analysis of the human ubiquitin-related proteome. Proteomics. 5:4145-4151. 